\title{
The Method of Determining the Angular Coordinates of Objects by Electro-Optical Means on the Basis of Space-Time Processing
}

\author{
Alexander Ju. Koziratsky, Alexander I. Grevtsev, \\ Vladimir V. Kapitanov and Anton A. Koziratsky* \\ Military Education and Research Centre of Military-Air Forces \\ «Military-Air Academy \\ Named After Professor N.E. Zhukovsky and Yu.A. Gagarin» \\ 54a Starykh Bolshevikov Str., Voronezh, 394064, Russia
}

Received 07.09.2016, received in revised form 27.12.2016, accepted 13.01.2017

Work using spatiotemporal image processing the developed method of determining the angular coordinates of the object, allowing to eliminate the influence of errors of positioning of the mechanical part of the optical system on the accuracy of measurement of angular coordinates.

Keywords: laser radar station; photodetector; element of photodetector; image; the angular coor dinates.

Citation: Koziratsky A.Ju., Grevtsev A.I., Kapitanov V.V., Koziratsky A.A. The method of determining the angular coordinates of objects by electro-optical means on the basis of space-time processing, J. Sib. Fed. Univ. Eng. technol., 2017, 10(2), $230-234$. DOI: 10.17516/1999-494X-2017-10-2-230-234.

(C) Siberian Federal University. All rights reserved

* Corresponding author E-mail address: kvv256@rambler.ru 


\title{
Способ определения угловых координат \\ объектов оптико-электронными средствами \\ на основе пространственно-временной обработки
}

\author{
А.Ю. Козирацкий, А.И. Гревцев, \\ В.В. Капитанов, А.А. Козирацкий \\ Военный учебно-научный цеентр Военно-воздушных сил \\ «Военно-воздушная академия \\ имени профессора Н.Е. Жуковского и Ю.А. Гагарина» \\ Россия, 394064, Воронеж, ул. Старых Большевиков, 54 а
}

С использованием пространственно-временной обработки изображений найден способ определения угловых координат объекта, позволяющий исключить влияние ошибок позиционирования механической части оптической системы на точность измерения угловых координат.

Ключевые слова: лазерная локачионная станция, фотоприемник, элемент фотоприемника, изображение, угловые координаты.

В настоящее время при определении угловых координат объектов широко используются оптико-электронные средства активной локации, которые состоят из лазерного передающего устройства, приемника оптического излучения, подсистем анализа и управления. Лазерная локационная станция осуществляет сканирование пространства по углу места и азимута за счёт механического перемещения элементов локатора. По величине этого перемещения определяются угловые координаты. В этом случае среднеквадратические ошибки углов измерения существенно зависят от конструкции механической части оптической системы [1].

Для повышения точности определения угловых координат необходимо использовать принципиально новые способы, основанные на пространственно-временной обработке регистрируемых изображений. Предпосылками использования таких способов являются существенное развитие фотоприемных матричных приборов, а также использование методов цифровой обработки сигналов [2] и существующих алгоритмов повышения разрешения [3].

Таким образом, целью статьи является разработка способа определения угловых координат объектов оптико-электронными средствами на основе пространственно-временной обработки последовательности получаемых изображений.

Суть способа заключается в следующем. Лазерная локационная станция сканирует сектор обзора, при этом передающий канал работает в импульсном режиме. В качестве приемной части лазерной локационной станции будем использовать матричный фотоприемник, который, регистрируя отраженное излучение, формирует изображение участка обозреваемого пространства.

От кадра к кадру средство локации перемещает свое поле зрения, два последовательно сформированных изображения будут иметь прямоугольную общую часть, размеры и положение которой пропорциональны изменению углового положения передающего канала. Область перекрытия соседних изображений в двумерной координатной плоскости представлена на рис. 1 , где $d$ и $h$ - размеры матричного фотоприемника; $\Delta y, \Delta x$ - размеры области перекрытия. 


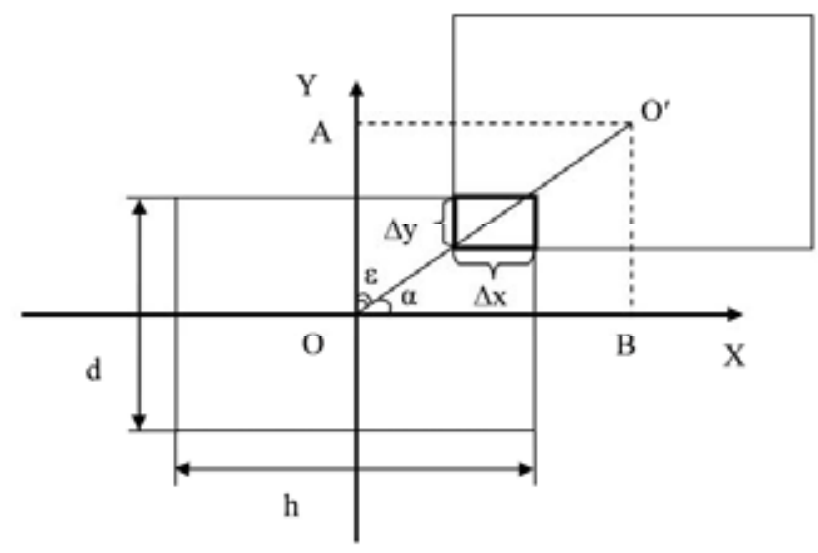

Рис. 1. Геометрия задачи

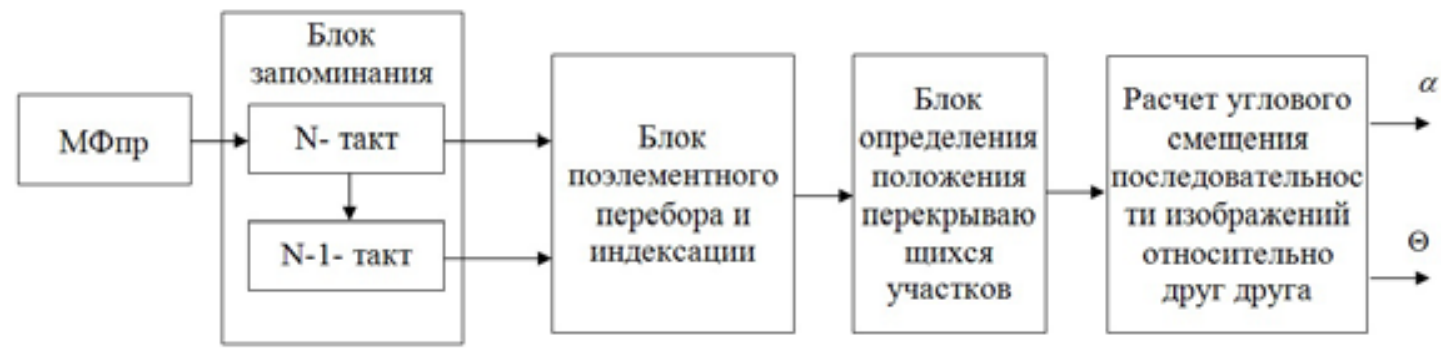

Рис. 2. Вычислительная схема

Для определения углового смещения последовательности изображений относительно друг друга разработана вычислительная схема пространственно-временной обработки двух изображений, показанная на рис. 2.

Схема работает следующим образом. С выхода матричного фотоприемника на каждом такте получаем матрицу яркостей, соответствующих получаемому изображению. Блок запоминания осуществляет запись в память текущей матрицы яркостей и хранение полученной на предыдущем шаге. После записи данных в память в процессе получения следующего кадра изображения происходит определение углового перемещения текущего кадра относительного предыдущего. Для этого блок поэлементного перебора и индексации осуществляет поэлементное сравнение текущей матрицы $m_{i, j}$ со всеми элементами предыдущей $n_{x, y}$ и исследуется их сходство. Учитывая, что полного совпадения элементов участка перекрытия, как правило, не бывает из-за шумов и искажений, введем в рассмотрение некую меру различения, которая показывает допустимый уровень похожести элементов по формуле

$$
\varepsilon\left(m_{i, j}, n_{x, y}\right)<L_{e},
$$

где $L_{e}-$ некоторый порог, зависящий от интенсивности шумов и чувствительности элементов матричного фотоприемника. 
Далее осуществляется процедура бинаризации, где при выполнении на текущем шаге условия (1) элементу $m_{i, j}$ присваивам значение «единица», а по окончании сравнения элементам, не удовлетворившим условию (1), присваиваем «ноль».

Полученная матрица, элементы которой имеют нулевые и единичные значения, поступает в блок определения положения перекрывающихся участков. Данный блок осуществляет нахождение значений координат ячеек через определение положения единичных строк и столбцов. Условием нахождения перекрывающейся области считается наличие четырех единичных ячеек в виде локальной матрицы размерностью $2 \times 2$ элемента.

Полученные значения координат элементов матрицы, являющиеся вершинами прямоугольной области перекрытия, поступают в блок расчета углового смещения последовательности изображений относительно друг друга.

Таким образом, вычислительная схема позволяет в двумерной системе координат ХОY текущего изображения определить размеры зоны перекрытия в виде координат начала и конца соответствующих строк и столбцов, по которым, с учетом проекций на соответствующие координатные оси ОХ и ОY, определяют азимут и угол места на объект по следующим формулам:

$$
\begin{aligned}
& \operatorname{tg} \alpha=\frac{B O^{\prime}}{O B}=\frac{d-\Delta y}{h-\Delta x}, \\
& \operatorname{tg} \varepsilon=\frac{A O^{\prime}}{O A}=\frac{h-\Delta x}{d-\Delta y} .
\end{aligned}
$$

Размеры области перекрытия рассчитывают выражениями:

$$
\begin{gathered}
\Delta x=\left(X_{\text {тек ,нач }}-X_{\text {тек ,кон }}+1\right) h_{x}, \\
\Delta y=\left(Y_{\text {нач,тек }}-Y_{\text {кон,тек }}+1\right) h_{y},
\end{gathered}
$$

где $X_{\text {тек,нач }}, X_{\text {тек,кон }}, Y_{\text {нач,тек }}$ и $Y_{\text {кон,тек }}$ - номера элементарных фотоприемников, соответствующие началу и окончанию области перекрытия по двум осям. Текущее значение номера фотоприемника, с учетом прямоугольности области перекрытия, выбирается как среднее между крайними точками.

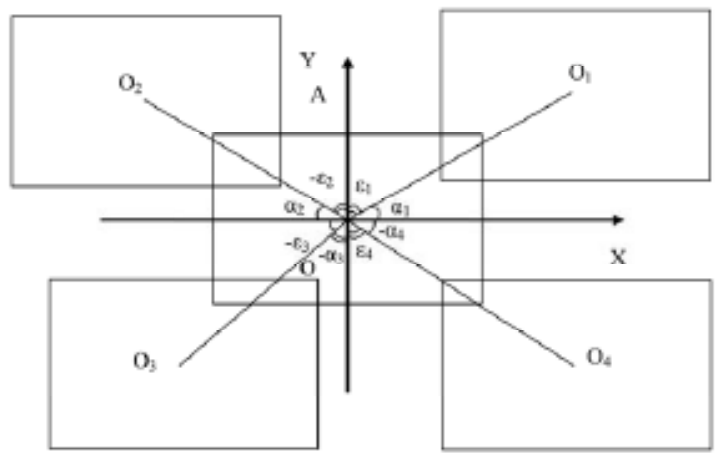

Рис. 3. Выбор знака углов азимута и места на объект 
Выбор знака углов $\alpha$ и $\varepsilon$ осуществляется исходя из четверти сравниваемых изображений исходной системы координат, как показано на рис. 3. Номер четверти определяется исходя из полученных координат перекрывающейся области текущего изображения.

Таким образом, на основе пространственно-временной обработки последовательности регистрируемых изображений разработан способ, позволяющий определять угловые координаты объекта и исключающий влияние ошибок позиционирования механической части оптической системы на точность измерения угловых координат.

\section{Список литературы}

[1] Краснов А.М. Оптико-электронные системы авиаџионного вооружения. М.: ВВИА им. проф. Н.Е. Жуковского, 2007. 1272 с. [Krasnov А. М. Optical-electronic systems of aircraft armament. Moscow, VVIA im. prof. E. Zhukovsky, 2007, 1272 p. (in Russian)].

[2] Гонсалес Р., Вудс Р. Цифровая обработка изображений. М: Техносфера, 2005. 1072 c. [Gonzalez R., Woods R. Digital image processing. Moscow, Technosphere, 2005, 1072 p. (in Russian)].

[3] Козирацкий А.Ю., Гревцев А.И., Фролов М.М. Способ повышения разрешения оптических изображений. Радиотехника. Информационный конфликт в спектре электромагнитных волн, 2015, 12, 54-59 [Kozirazki A. Yu., Grevtsev A. I., Frolov M. M. Method of increasing resolution of optical images. Radiotec. Information conflict in the spectrum of electromagnetic waves, 2015, 12, 54-59 (in Russian)]. 